\title{
A retrospective analysis of patient-specific factors on voriconazole clearance
}

\author{
Satoshi Dote ${ }^{1 *}$, Maki Sawai ${ }^{1}$, Ayumu Nozaki ${ }^{1}$, Kazumasa Naruhashi $^{2}$, Yuka Kobayashi $^{1}$ and Hirokazu Nakanishi ${ }^{2}$
}

\begin{abstract}
Background: Voriconazole concentrations display a large variability, which cannot completely be explained by known factors. We investigated the relationships of voriconazole concentration with patient-specific variables and concomitant medication to identify clinical factors affecting voriconazole clearance.

Methods: A retrospective chart review of voriconazole trough concentration, laboratory data, and concomitant medication in patients was performed. The concentration/dose ratio (C/D-ratio) was assessed as a surrogate marker of total clearance by dividing voriconazole concentration by daily dose per $\mathrm{kg}$ of body weight.

Results: A total of 77 samples from 63 patients were obtained. In multiple linear regression analysis, increased C-reactive protein (CRP) level $(p<0.05)$ and decreased albumin (Alb) level $(p<0.05)$ were associated with significantly increased C/D-ratio of voriconazole, and coadministration with a glucocorticoid was associated with significantly $(p<0.05)$ decreased C/D-ratio of voriconazole (adjusted $r^{2}=0.31$ ). Regarding CRP and Alb, receiver operating characteristic curve analysis indicated that increased CRP level and decreased Alb level were significant predictors of toxic trough concentration of voriconazole. For CRP, area under the curve (AUC) and cutoff value were 0.71 (95\% confidence interval $(\mathrm{Cl}), 0.57-0.86, p<0.01$ ) and $4.7 \mathrm{mg} / \mathrm{dl}$, respectively. For Alb, AUC and cutoff value were $0.68(95 \% \mathrm{Cl}, 0.53-0.82, p<0.05)$ and $2.7 \mathrm{~g} / \mathrm{dl}$, respectively. A significant difference was seen in voriconazole trough concentration between patients with hepatotoxicity and those without $(5.69 \mu \mathrm{g} / \mathrm{ml} \mathrm{vs} 3.0 \mu \mathrm{g} / \mathrm{ml}, p<0.001)$.
\end{abstract}

Conclusion: Coadministration of glucocorticoid and inflammation, reflected by elevated CRP level and hypoalbuminemia, are associated with voriconazole clearance. We propose that early measurement of voriconazole concentration before the plateau phase will lead to avoidance of a toxic voriconazole level in patients with elevated CRP level and hypoalbuminemia, although further studies are needed to confirm our findings.

Keywords: Voriconazole, Pharmacokinetics, Therapeutic drug monitoring, Drug interaction, Inflammation

\section{Background}

The triazole antifungal voriconazole is widely used in the treatment of invasive fungal infections, such as invasive aspergillosis, candidemia, and pulmonary cryptococcosis, except for zygomycosis [1-4]. Voriconazole exhibits a nonlinear pharmacokinetics profile and is metabolized primarily by the cytochrome P450 family enzyme CYP2C19 and, to lesser extent, CYP3A4 and CYP2C9 [1-4]. Allelic polymorphisms of CYP2C19 have been shown to be the most important determinants of the clearance efficiency of vorizonazole $[3,5]$. In Japanese, the rate of poor metabolizers of CYP2C19 has been shown to be $15-20 \%$,

\footnotetext{
* Correspondence: everyday.is.a.new.day.1981@gmail.com

'Department of Pharmacy, Kyoto-Katsura Hospital, 17 Yamadahirao-cho,

Nishikyo-ku, Kyoto 615-8256, Japan

Full list of author information is available at the end of the article
}

a higher figure than that of other races [6]. This raises the necessity of blood concentration measurement for Japanese to monitor efficacy and toxicity in the clinical setting. There have been some reports on the relationships between efficacy and toxicity of voriconazole and its plasma concentrations. Target trough concentration of therapeutic response is $>1-2 \mu \mathrm{g} / \mathrm{ml}[1,3,4,7-9]$, and prophylaxis is $>1.5 \mu \mathrm{g} / \mathrm{ml}$ [3]. To avoid side effects, such as visual disturbance, neurotoxicity, and hepatotoxicity, the target trough concentration is $<4-6 \mu \mathrm{g} / \mathrm{ml}$ $[1,3,4,7-10]$. Invasive fungal infections commonly appear among immunocompromised patients receiving concomitant medication for primary disease and/or bacterial infection. Many of them have critically ill conditions and polypharmacy, which possibly alter voriconazole 
concentration and clearance. Accordingly, to identify clinical factors affecting voriconazole clearance, we investigated the relationships of voriconazole concentration with patient-specific variables and concomitant medication.

\section{Methods}

\section{Patient enrollment and data collection}

Patients who received voriconazole orally and had at least one voriconazole trough concentration measurement during therapy at Kyoto-Katsura Hospital, Japan, between June 2010 and October 2015 were eligible for inclusion. Patient-specific variables were collected retrospectively including sex, age, body weight, body mass index (BMI), laboratory data, purpose of voriconazole administration, and diagnosis and treatment department. Laboratory data included serum albumin (Alb), creatinine clearance (Ccr), aspartate aminotransferase (AST), alanine aminotransferase (ALT), total bilirubin (T-Bil), alkaline phosphatase (ALP), $\gamma$-glutamyl transpeptitase ( $\gamma$ GTP), and $C$-reactive protein (CRP). Ccr was estimated by using the CockcroftGault Formula [11]. Laboratory data at voriconazole trough concentration measurement were used for analysis. Daily dose, voriconazole trough concentration, the number of days between blood sample collection and initial dose were collected. Patients were excluded if: (a) they received voriconazole intravenously even once to avoid unknown covariates between orally and intravenously; (b) they received concomitant rifabutin, rifampicin, carbamazepine, phenytoin, or antiretroviral drugs; (c) they required dialysis since the uremic substances, which should accumulate in the blood of those patients, may alter the pharmacokinetics of voriconazole; or (d) the number of days between blood sample collection and initial voriconazole administration with loading dose is 2 or less, or that without loading dose is 4 or less, because the concentration did not reach a steady state $[3,4]$. Affect of concomitant glucocorticoid (over $5 \mathrm{mg}$ equivalent prednisolone as a daily dose) and macrolide was analyzed in all patients, because those medications can interact with voriconazole pharmacokinetically $[9,12,13]$. Cases in which the voriconazole dose was changed, or the above-mentioned concomitant medications were started or discontinued, were regarded as separate cases. Visual disturbance such as photophobia, blurred vision, neurotoxicity such as drowsiness, insomnia, hallucinations, disturbance of consciousness, and hepatotoxicity were evaluated as adverse events. Hepatotoxicity was defined as $\geq$ grade 2 in results of liver function tests (AST, ALT, T-Bil, ALP, and $\gamma$ GTP) within 14 days after initiation of voriconazole administration or changing voriconazole dosage, according to Common Terminology Criteria for Adverse Events (CTCAE), version 4.0, since some of ALP and $\gamma$ GTP values in the patients were over normal range (about grade 1) before the voriconazole therapy. We used voriconazole concentration at the time of occurrence of toxicity in the patients with adverse event, whereas the maximum concentration in the patients without adverse event for the analysis of relationship between adverse events and voriconazole concentration. All voriconazole concentration data were collected from a central referral laboratory (SRL Inc., Tokyo, Japan). A validated highperformance liquid chromatography (HPLC) assay was used to measure voriconazole concentrations [14].

The study was performed in accordance with the Declaration of Helsinki and its amendments, and the protocol was approved by the Ethics Committee of Kyoto-Katsura Hospital.

\section{Analysis of voriconazole pharmacokinetics}

The concentration/dose ratio (C/D-ratio) was used as a surrogate marker of total clearance by dividing voriconazole concentration by daily dose per $\mathrm{kg}$ of body weight.

\section{Statistical analysis}

Univariate linear regression analysis was used to estimate relationships between C/D-ratio of voriconazole and patient-specific continuous variables, and the unpaired $t$ test was used to compare voriconazole concentration and C/D-ratio of voriconazole between two independent groups. Multiple linear regression analysis was applied to identify factors that contribute to the variability in C/D-ratio of voriconazole; a step-down selection model was obtained using stepwise regression with a $p$ value of 0.25 as the inclusion criterion. The diagnostic properties and cutoff values of independent variables by multiple linear regression analysis were cross-sectionally evaluated by a receiver operating characteristic (ROC) curve. Outcome for diagnostic properties was voriconazole trough concentration exceeding $4 \mu \mathrm{g} / \mathrm{ml}$, defined as a toxic level according to the results of meta-analysis by Hamada et al. [8]. JMP ${ }^{\circ} 9$ software was used for all analyses and a $p$ value less than 0.05 was regarded as statistically significant.

\section{Results \\ Patient characteristics}

A total of 77 samples from 63 patients were obtained in the present study (Table 1). Eligible patients were elderly, with a mean age of 70.8. Mean daily maintenance dose and mean body weight were $273 \mathrm{mg}$ and $49.7 \mathrm{~kg}$, respectively. The mean dosage is close to, but slightly lower than the recommend daily oral dose on the package insert [15] for patients with a body weight $\geq 40 \mathrm{~kg}(300-400 \mathrm{mg})$. This may be caused by the suggestion by the clinical pharmacist to the prescription doctors to reduce the voriconazole dosage, since the pharmacist had experienced higher concentration of voriconazole in patients with higher CRP values, empirically. Twentyeight patients received glucocorticoid as a concomitant 
Table 1 Patient characteristics

\begin{tabular}{|c|c|}
\hline & Mean (SD) \\
\hline Male/Female & $50 / 13$ \\
\hline Age (yr) & $70.8(11.0)$ \\
\hline Body Weight (kg) & $49.7(9.5)$ \\
\hline Body Mass Index (kg/m²) & $19.0(2.9)$ \\
\hline Albumin $(g / d l)^{a}$ & $2.7(0.7)$ \\
\hline Creatinine clearance $(\mathrm{ml} / \mathrm{min})^{a}$ & $67.4(31.9)$ \\
\hline AST $(I U / L)^{a}$ & $32.9(28.6)$ \\
\hline $\mathrm{ALT}(\mathrm{IU} / \mathrm{L})^{\mathrm{a}}$ & $32.9(35.9)$ \\
\hline $\operatorname{ALP}(I U / L)^{a}$ & $386.6(409.2)$ \\
\hline$\gamma G T P(I U / L)^{a}$ & $103.7(173.1)$ \\
\hline T-Bil $(\mathrm{mg} / \mathrm{dl})^{\mathrm{a}}$ & $0.6(0.8)$ \\
\hline$C$ reactive protein $(\mathrm{mg} / \mathrm{dl})^{\mathrm{a}}$ & $5.6(6.4)$ \\
\hline Daily dose $(\mathrm{mg} / \text { day) })^{a}$ & $273(89.8)$ \\
\hline Trough concentration $(\mu \mathrm{g} / \mathrm{ml})^{\mathrm{a}}$ & $3.2(2.3)$ \\
\hline \multicolumn{2}{|l|}{ Trough concentration collection date (after initial dose) ${ }^{a}$} \\
\hline with loading dose & $9.7(13)$ \\
\hline without loading dose & $11.1(9.5)$ \\
\hline $\begin{array}{l}\text { Purpose of voriconazole administration } \\
\text { prophylaxis/treatment }\end{array}$ & $3 / 60$ \\
\hline \multicolumn{2}{|l|}{ Diagnosis and treatment department } \\
\hline Hematology/Pulmonology/Rheumatology/Other & $41 / 17 / 4 / 1$ \\
\hline \multicolumn{2}{|l|}{ Concomitant medication $^{a}$} \\
\hline Glucocorcicoid & 28 \\
\hline Macrolide Antibiotics & 2 \\
\hline
\end{tabular}

${ }^{\mathrm{a} A}$ cumulative total of 77 samples

These data were collected at the time of initial voriconazole administration

medication and most of them (23. $82 \%)$ received prednisolone.

\section{Factors affecting voriconazole concentration and clearance}

Significant correlation was found between administered dosage and trough concentration of voriconazole $\left(r^{2}=0.14, p<0.001\right)$, (Fig. 1). Mean (SD) C/Dratio significantly decreased from $0.66(0.42)$ to 0.43 $(0.33)$ by concomitant administration with glucocorticoid ( $p=0.01)$ (Fig. 2). Effect of concomitant macrolide on C/D-ratio was not conducted because only two patients was administered erythromycin. Significant correlations were found between C/D-ratio of voriconazole and Alb $\left(r^{2}=0.16, p<0.01\right)$, CRP $\left(r^{2}=0.26, p<0.0001\right)$. Alb, CRP, and age $(p=0.07)$ were treated as continuous variables and glucocorticoid use was treated as a dichotomous variable in multiple linear regression analysis. Increased CRP level and decreased Alb level were significantly associated with increased C/D-ratio of voriconazole, and coadministration with a glucocorticoid was significantly associated with decreased C/D-ratio of voriconazole

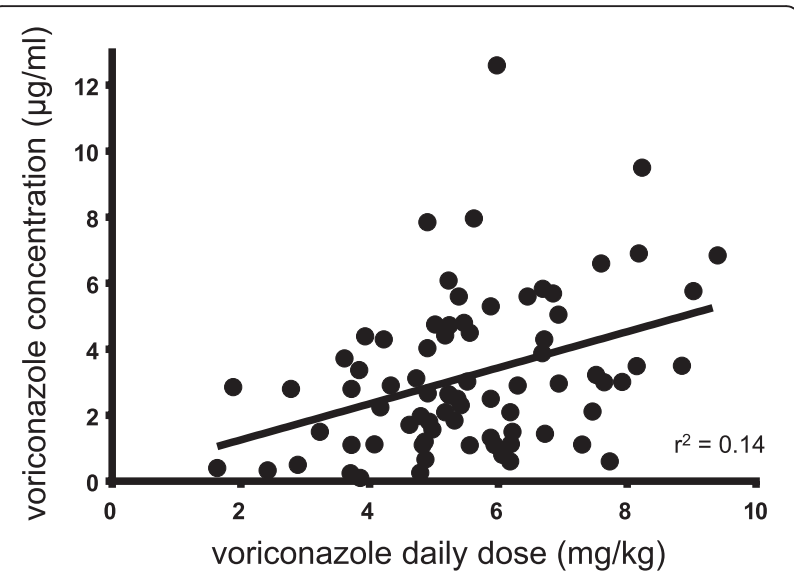

Fig. 1 Relationship between voriconazole dosages per patient weight and voriconazole trough concentration. Each point represents a measurement. The linear regression curve is presented with coefficient of determination $\left(r^{2}\right)$

(adjusted $r^{2}=0.31$ ) (Table 2). We did a post-hoc analysis of the effect of coadministration with a glucocorticoid on interpatient variability of voriconazole concentration in three patients (Table 3). C/D-ratio was considerably decreased by glucocorticoid administration in all three patients.

\section{Cutoff values of factors causing toxic level of voriconazole concentration}

Multiple linear regression analysis indicated that CRP and Alb were the factors affecting voriconazole clearance. ROC curve analysis indicated that increased CRP level and decreased Alb level were significant predictors of toxic trough concentration of voriconazole: A) CRP - area under the curve (AUC) 0.71 (95\% confidence interval [CI], 0.57-0.86, $p<0.01$ ), cutoff value $4.7 \mathrm{mg} / \mathrm{dl}$ (sensitivity $60.9 \%$, specificity $82.7 \%$ ); B) Alb - AUC 0.68 (95\% CI,

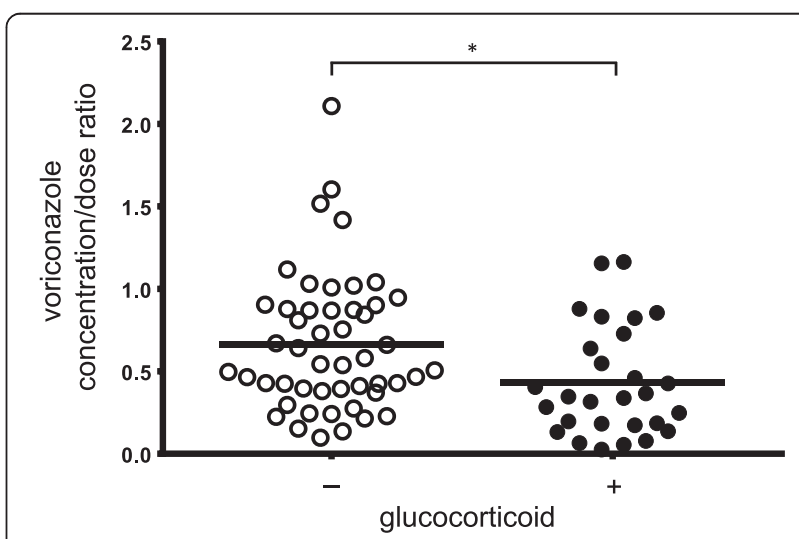

Fig. 2 Comparison of mean voriconazole concentration/dose ratio. A scatter plot of voriconazole concentration/dose ratio from 77 samples for 63 patients without coadministration of glucocorticoid $(n=49)$ and with coadministration of glucocorticoid $(n=28)$. Solid line means mean 
Table 2 Factors associated with a significant change in vorizconazole clearance identified from multiple linear regression analysis

\begin{tabular}{|c|c|c|c|c|c|}
\hline \multirow[b]{2}{*}{ Model term } & \multirow[b]{2}{*}{ Coefficient } & \multicolumn{2}{|c|}{95 \% Confidence interval } & \multirow[b]{2}{*}{ Variance inflation factors } & \multirow[b]{2}{*}{$p$ value } \\
\hline & & Lower & Upper & & \\
\hline Albumin (g/dl) & -0.19 & -0.36 & -0.03 & 1.55 & $<0.05$ \\
\hline C reactive protein (mg/dl) & 0.02 & 0.004 & 0.04 & 1.51 & $<0.05$ \\
\hline Glucocorticoid administration & -0.25 & -0.45 & -0.05 & 1.18 & $<0.05$ \\
\hline
\end{tabular}

$0.53-0.82, p<0.05)$, cutoff value $2.7 \mathrm{~g} / \mathrm{dl}$ (sensitivity $61.9 \%$, specificity $73.7 \%$ ) (Fig. 3).

\section{Adverse effects}

Incidence of hepatotoxicity, visual disturbance, and neurotoxicity were $20.3,43.5$, and $22.6 \%$, respectively. Twelve episodes of hepatotoxicity were observed: grade 2, six; grade 3, six. Summary of hepatotoxicity were: grade 2 elevation of ALT, one; of ALP, two; of $\gamma$ GTP, four; grade 3 elevation of ALT, one; of ALP, two; of $\gamma \mathrm{GTP}$, six. The voriconazole trough concentration were significantly higher in patients with grade 2-3 hepatotoxicity than in patients without, whereas no significant difference was seen in visual disturbance and neurotoxicity (Fig. 4).

\section{Discussion}

The results of the present study have shown that CRP elevation and hypoalbuminemia decreased voriconazole clearance and that concomitant glucocorticoid administration increased voriconazole clearance. In multiple linear regression analysis, we did not detect the presence of multicolinearity between CRP and glucocorticoid administration (variance inflation factors $<2$ ), suggesting that CRP and concomitant glucocorticoid administration should be independent of each other in affecting voriconazole clearance. Although these two factors are independent, we speculate these factors might be associated with alteration of metabolism of voriconazole; elimination of voriconazole is mainly by metabolism of CYP2C19 dominantly and of CYP3A4 and CYP2C9 being involved to a much lesser extent [1-4].

Michael et al. retrospectively investigated therapeutic drug monitoring of voriconazole in a cohort of 201 patients [9]. They identified by multiple linear regression analysis that the use of a systemic glucocorticoid was associated with significantly reduced voriconazole blood concentrations. They hypothesized that glucocorticoid up-regulated CYP2C19 and CYP3A4 expression since some in vitro $[16,17]$ and in vivo $[18,19]$ studies have shown that glucocorticoid can bind to and activate the pregnane $\mathrm{X}$ receptor, one of the nuclear receptors which plays a role in inducing cytochrome P450 expression, including $\mathrm{CYP} 2 \mathrm{C} 19$ and $\mathrm{CYP} 3 \mathrm{~A} 4$, resulting in the increase of metabolism by the enzymes.

Another study has shown that voriconazole clearance significantly decreased in a limited number of patients with CYP2C19 poor metabolizer and heterozygous extensive metabolizer when coadministered with erythromycin, a known CYP3A4 inhibitor [12]. In our study, of the three patients who administered concomitant glucocorticoid, $\mathrm{C} / \mathrm{D}$-ratio of one patient was considerably increased by discontinuation of glucocorticoid (Table 3, Case 1), and C/D-ratios of the two patients were considerably decreased by start of glucocorticoid (Table 3, Cases 2 and 3). They might be poor metabolizer of CYP2C19, resulting in the prominent increase of voriconazole metabolism due to CYP3A4 induction, although we did not perform the allele analysis of any gene of the patients.

Zordoky et al. reported that nuclear factor- $\mathrm{kB}$ plays a crucial role in the regulation of cytochrome $\mathrm{P} 450$ through several mechanisms and this role can explain the altered cytochrome P450 regulation in many conditions [20]. They suggested that in inflammation status, nuclear factor-kB-repressing factor is activated and cytochrome P450 expression is decreased. A significant decrease in voriconazole clearance observed in patients with elevated CRP level in our study. Other studies reported the inflammation is associated with higher voriconazole trough concentrations [13, 21], congruent with our results. Therefore, higher voriconazole trough concentrations in inflammatory patients might be related

Table 3 Change of voriconazole concentration and clearance about the patient with and without glucocorticoid administration

\begin{tabular}{|c|c|c|c|c|c|c|}
\hline \multirow[t]{2}{*}{ Patient } & \multicolumn{2}{|l|}{ Case. 1} & \multicolumn{2}{|c|}{ Case. 2} & \multicolumn{2}{|c|}{ Case. 3} \\
\hline & Pre & Post & Pre & Post & Pre & Post \\
\hline Glucocorticoid & mPSL 62.5 mg bid & - & - & DEX $20 \mathrm{mg}$ & - & PSL $20 \mathrm{mg}$ \\
\hline Daily dose (mg/day) & 300 & 300 & 200 & 150 & 400 & 200 \\
\hline Trough concentration ( $\mu \mathrm{g} / \mathrm{ml})$ & 4.03 & 7.85 & 3.37 & 0.5 & 3.22 & 0.1 \\
\hline C/D-ratio & 0.82 & 1.6 & 0.88 & 0.17 & 0.43 & 0.03 \\
\hline
\end{tabular}

Abbreviations: mPSL methylprednisolone, DEX dexamethasone, PSL prednisolone, bid twice daily 


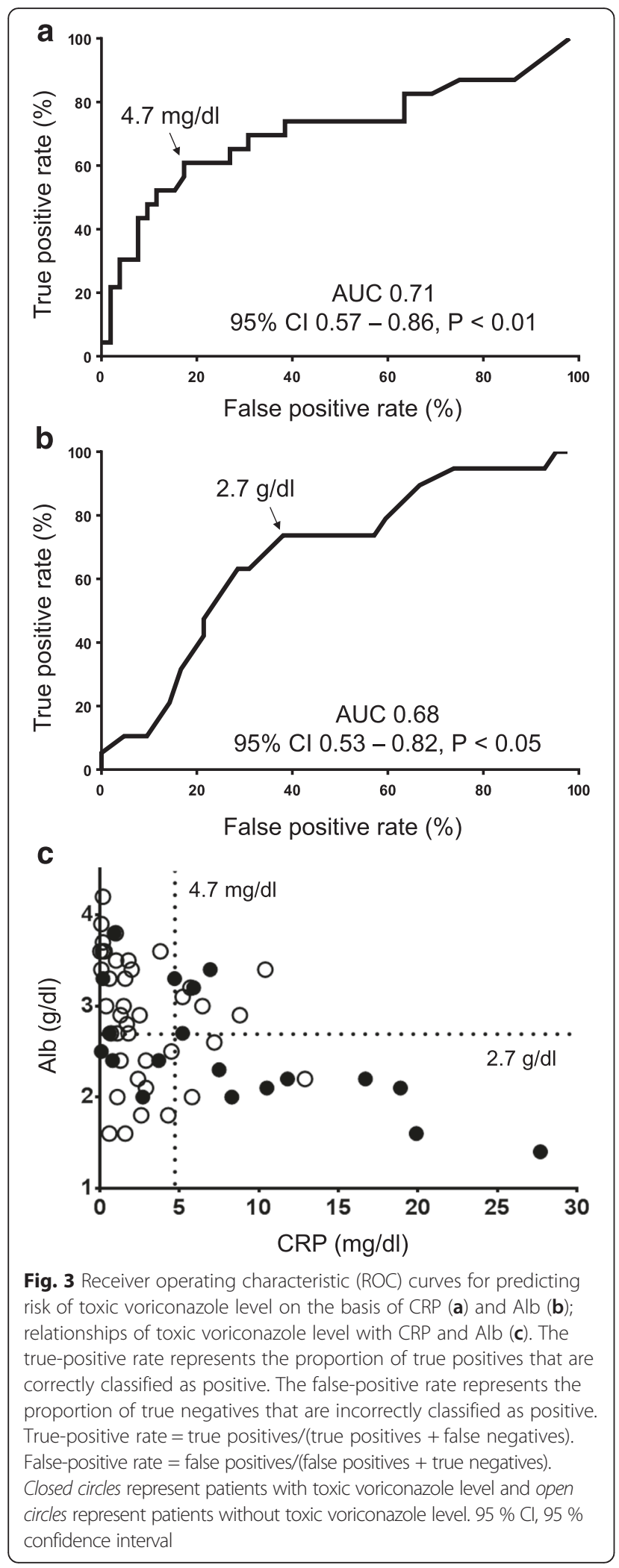

to the above mechanism, since elevated CRP level and hypoalbuminemia are regarded as markers for inflammatory status.

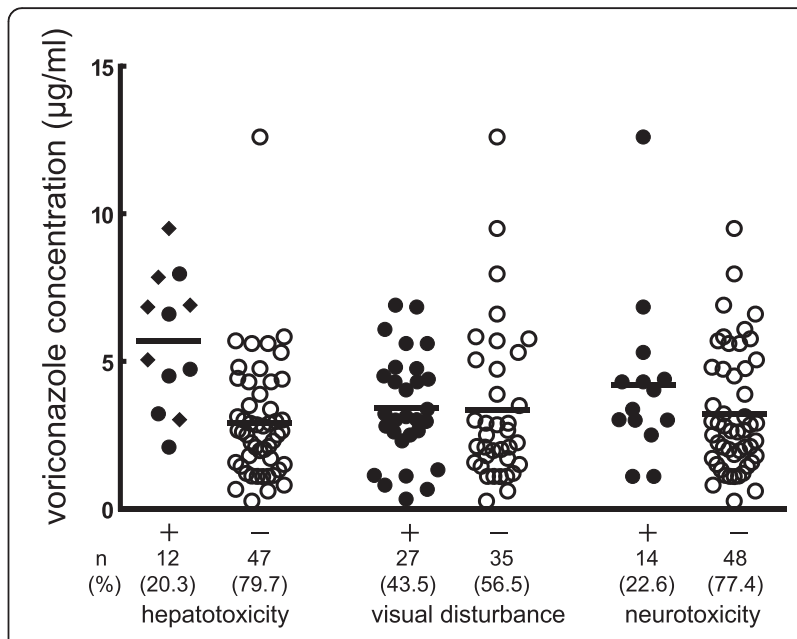

Fig. 4 Relationships between voriconazole concentration and toxicities. A scatter plot of voriconazole concentrations from all 63 patients. Closed circles represent patients with toxicity and open circles represent patients without toxicity. Closed diamond represents patients with Grade 3 hepatotoxicity. Solid line means mean. One patient was not assessed for visual disturbance and neurotoxicity because of the administration of a sedative. Four patients were not assessed for hepatotoxicity because of liver dysfunction due to primary disease. A significant difference was seen in voriconazole mean trough concentration between patients with grade 2-3 hepatotoxicity and those without: 5.69 (SD 2.27) vs. 3.0 (SD $2.07), p<0.001$. No significant difference was seen in voriconazole mean trough concentration between patients with other adverse events and those without: visual disturbance - 3.48 (SD 1.79) vs. 3.44 (SD 2.7), $p=0.94$; neurotoxicity -4.2 (SD 2.85) vs. 3.27 (SD 2.11), $p=0.18$

In addition, we undertook an ROC analysis for prediction of risk of toxic voriconazole level on the basis of CRP and Alb. The results suggest that CRP and Alb should be useful for screening of toxic voriconazole level. In particular, in most cases, voriconazole concentrations reached toxic levels when the CRP and Alb values of the patients were over the cutoff values described below (Fig. 3c). Consequently, careful monitoring of voriconazole concentration and side effects are required for patients with both elevated CRP level and hypoalbuminemia. From our analysis, the cutoff values of CRP and Alb were 4.7 and 2.7 respectively. Statistically, these cutoff values are useful for prediction of toxic voriconazole level, although the value of 4.7 of CRP is less clinically important, since the value is common level of infectious diseases. Voriconazole concentrations in patients with hepatotoxicity were significantly higher than those without. In addition, the concentrations of voriconazole in $75 \%$ of patients with hepatotoxicity were over $4 \mu \mathrm{g} / \mathrm{ml}$. This result suggests that voriconazole trough concentration over $4 \mu \mathrm{g} / \mathrm{ml}$ should be avoided, supported by previous reports $[8,10]$.

A limitation of the present study is that CYP2C19 polymorphism was not available. However, Geist et al. found an enormous (88\%) interpatient variability in 
voriconazole trough concentrations, although none of the patients were classified as a CYP2C19 poor metabolizer. They noted that the high variability resulted from contributing factors, including changes in drug absorption, co-medication, and disease status [22]. Further studies are needed to clarify contributing factors in patients with known genetic polymorphisms of the cytochrome P450 isoenzymes.

\section{Conclusion}

We evaluated patient-specific factors affecting voriconazole clearance and showed that increased CRP level and decreased Alb level were significant predictors of decreased voriconazole clearance, and coadministration of glucocorticoid was found to be a significant predictor of increased voriconazole clearance. It is not practical to examine CYP2C19 alleles due to the cost, so it is difficult to consider CYP2C19 polymorphism in therapeutic drug monitoring in routine practice. Therefore, we propose that early measurement of voriconazole concentration before the plateau phase should lead to the avoidance of toxic voriconazole level in patients with elevated CRP level and hypoalbuminemia.

\section{Competing interests}

The authors declare that they have no competing interests.

\section{Authors' contributions}

Conception and design of this study, analysis, and interpretation of data were performed by SD. SD and MS carried out assessment of voriconazole toxicities. Critical revision of this article for important intellectual content was performed by $A N, K N$ and $Y K$. HN reviewed and provided final approval of submission of this article. All authors read and approved the final manuscript

\section{Acknowledgement}

None declared.

\section{Author details}

1 Department of Pharmacy, Kyoto-Katsura Hospital, 17 Yamadahirao-cho, Nishikyo-ku, Kyoto 615-8256, Japan. Faculty of Pharmaceutical Sciences, Doshisha Women's College of Liberal Arts, Kodo, Kyotanabe-shi, Kyoto 610-0395, Japan.

Received: 18 December 2015 Accepted: 28 March 2016

Published online: 19 April 2016

\section{References}

1. Dolton MJ, McLachlan AJ. Optimizing azole antifungal therapy in the prophylaxis and treatment of fungal infections. Curr Opin Infect Dis. 2014; 27:493-500.

2. Traunmuller F, Popovic M, Konz KH, Smolle-Juttner FM, Joukhadar C. Efficacy and safety of current drug therapies for invasive aspergillosis. Pharmacology. 2011;88:213-24.

3. Seyedmousavi S, Mouton JW, Verweij PE, Bruggemann RJ. Therapeutic drug monitoring of voriconazole and posaconazole for invasive aspergillosis. Expert Rev Anti Infect Ther. 2013;11:931-41.

4. Hamada Y, Tokimatsu I, Mikamo H, Kimura M, Seki M, Takakura S, Ohmagari N, Takahashi Y, Kasahara K, Matsumoto K, et al. Practice guidelines for therapeutic drug monitoring of voriconazole: a consensus review of the Japanese Society of Chemotherapy and the Japanese Society of Therapeutic Drug Monitoring. J Infect Chemother. 2013;19:381-92.

5. Lee S, Kim BH, Nam WS, Yoon SH, Cho JY, Shin SG, Jang IJ, Yu KS. Effect of CYP2C19 polymorphism on the pharmacokinetics of voriconazole after single and multiple doses in healthy volunteers. J Clin Pharmacol. 2012;52: 195-203.

6. Shimizu T, Ochiai H, Asell F, Shimizu H, Saitoh R, Hama Y, Katada J, Hashimoto M, Matsui $H$, Taki K, et al. Bioinformatics research on inter-racial difference in drug metabolism I. Analysis on frequencies of mutant alleles and poor metabolizers on CYP2D6 and CYP2C19. Drug Metab Pharmacokinet. 2003;18:48-70.

7. Ueda K, Nannya Y, Kumano K, Hangaishi A, Takahashi T, Imai Y, Kurokawa M. Monitoring trough concentration of voriconazole is important to ensure successful antifungal therapy and to avoid hepatic damage in patients with hematological disorders. Int J Hematol. 2009;89:592-9.

8. Hamada Y, Seto Y, Yago K, Kuroyama M. Investigation and threshold of optimum blood concentration of voriconazole: a descriptive statistical metaanalysis. J Infect Chemother. 2012;18:501-7.

9. Dolton MJ, Ray JE, Chen SC, Ng K, Pont LG, McLachlan AJ. Multicenter study of voriconazole pharmacokinetics and therapeutic drug monitoring. Antimicrob Agents Chemother. 2012;56:4793-9.

10. Suzuki Y, Tokimatsu I, Sato Y, Kawasaki K, Goto T, Hashinaga K, Itoh H, Hiramatsu K, Kadota J. Association of sustained high plasma trough concentration of voriconazole with the incidence of hepatotoxicity. Clin Chim Acta. 2013;424:119-22.

11. Cockcroft DW, Gault MH. Prediction of creatinine clearance from serum creatinine. Nephron. 1976;16:31-41.

12. Shi HY, Yan J, Zhu WH, Yang GP, Tan ZR, Wu WH, Zhou G, Chen XP, Ouyang DS. Effects of erythromycin on voriconazole pharmacokinetics and association with CYP2C19 polymorphism. Eur J Clin Pharmacol. 2010:66:1131-6.

13. Naito T, Yamada T, Mino Y, Kawakami J. Impact of inflammation and concomitant glucocorticoid administration on plasma concentration of triazole antifungals in immunocompromised patients. Clin Chim Acta. 2015;441:127-32.

14. Usuki H, Inoue T, Ishida T, Miyazaki N. Determination of voriconazole by highperformance liquid chromatography. Igaku To Yakugaku. 2005;53:817-21.

15. VFEND ${ }^{\oplus}$ Tablets [package insert]. Pfizer Japan Inc., Tokyo, Japan; Revised October. 2014

16. Chen Y, Ferguson SS, Negishi M, Goldstein JA. Identification of constitutive androstane receptor and glucocorticoid receptor binding sites in the CYP2C19 promoter. Mol Pharmacol. 2003;64:316-24.

17. Martin P, Riley R, Back DJ, Owen A. Comparison of the induction profile for drug disposition proteins by typical nuclear receptor activators in human hepatic and intestinal cells. Br J Pharmacol. 2008;153:805-19.

18. Villikka K, Varis T, Backman JT, Neuvonen PJ, Kivisto KT. Effect of methylprednisolone on CYP3A4-mediated drug metabolism in vivo. Eur J Clin Pharmacol. 2001;57:457-60.

19. Shimada T, Terada A, Yokogawa K, Kaneko H, Nomura M, Kaji K, Kaneko S, Kobayashi K, Miyamoto K. Lowered blood concentration of tacrolimus and its recovery with changes in expression of CYP3A and P-glycoprotein after high-dose steroid therapy. Transplantation. 2002;74:1419-24.

20. Zordoky BN, El-Kadi AO. Role of NF-kappaB in the regulation of cytochrome P450 enzymes. Curr Drug Metab. 2009;10:164-78.

21. van Wanrooy MJ, Span LF, Rodgers MG, van den Heuvel ER, Uges DR, van der Werf TS, Kosterink JG, Alffenaar JW. Inflammation is associated with voriconazole trough concentrations. Antimicrob Agents Chemother. 2014; 58:7098-101.

22. Geist MJ, Egerer G, Burhenne J, Riedel KD, Weiss J, Mikus G. Steady-state pharmacokinetics and metabolism of voriconazole in patients. J Antimicrob Chemother. 2013:68:2592-9.

Submit your next manuscript to BioMed Central and we will help you at every step:

- We accept pre-submission inquiries

- Our selector tool helps you to find the most relevant journal

- We provide round the clock customer support

- Convenient online submission

- Thorough peer review

- Inclusion in PubMed and all major indexing services

- Maximum visibility for your research

Submit your manuscript at www.biomedcentral.com/submit 